\title{
Influencia de autoconcepto en los estilos de vida de estudiantes de enfermería en una universidad privada del Este de Lima
}

\author{
Influence of self-concept in the lifestyles of nursing students
}

in a private university in Eastern Lima

\author{
Mayela Cajachagua Castro ${ }^{1}$, Lindsey W. Vilca ${ }^{2}$ y Roussel Davila Villavicencio ${ }^{3}$ \\ ${ }^{1}$ Doctora en Educación. Magíster en Salud Pública. Licenciada en Enfermería. Docente \\ investigadora de la Facultad de Ciencias de la Salud, Universidad Peruana Unión, Lima, Perú. \\ E-mail: mayela@upeu.edu.pe \\ ${ }^{2}$ Psicólogo, egresado de la maestría en Psicología Clínica, mención en Neuropsicología. Docente \\ investigador en la Escuela Profesional de Psicología, Universidad Peruana Unión, Lima, Perú. \\ E-mail: lwvilca@upeu.edu.pe \\ ${ }^{3}$ Doctor en Ciencias de la Enfermería. Magíster en Enfermería. Licenciado en Enfermería. \\ Decano de la Facultad de Ciencias de la Salud, Universidad Peruana Unión, Lima, Perú. \\ E-mail: roussel@upeu.edu.pe \\ Universidad Peruana Unión, Lima, Perú
}

\section{Resumen}

La formación académica de los profesionales de ciencias de la salud lleva implícito el desarrollo armonioso e integral como ser humano, considerando, entre otros elementos, el autoconcepto y los estilos de vida a fin de que se constituya en un agente proactivo de cambio en el lugar donde se desempeñe. El objetivo de este estudio fue identificar la influencia del autoconcepto sobre los estilos de vida en estudiantes de una universidad privada de Lima Este. Su diseño es no experimental de tipo cuantitativo con enfoque explicativo-causal y transversal. El método de muestreo utilizado fue el no probabilístico. Participaron 135 estudiantes que cumplieron con los criterios de inclusión y exclusión y contó con la aprobación del Comité de Ética de la universidad. Para el logro de los objetivos, se aplicó el cuestionario de estilos de vida (PEPS-I), para la variable autoconcepto el test AF-5. En el análisis descriptivo, el promedio del perfil de estilos de vida corresponde a la dimensión autoactualización (43.2), seguido de la dimensión responsabilidad en la salud (26.1) y la dimensión ejercicio con el promedio más bajo (12.4). En el análisis de la variable autoconcepto, el autoconcepto social obtuvo mayor promedio (43.2) y el autoconcepto físico presentó menor promedio (28.2). Una vez concluido el estudio, se pudo afirmar que el autoconcepto influye significativamente sobre el estilo de vida de los estudiantes $(\beta=$ $.80 ; \mathrm{p}<.05)$; es decir que el autoconcepto logró explicar el $80 \%$ del perfil de estilo de vida que llevaban los estudiantes.

Palabras clave: autoconcepto, estilos de vida saludables, estudiantes de enfermería.

\section{Abstract}

The academic formation of health science professionals involves their harmonious and integral development as human beings, considering, among other elements, self-concept and lifestyles, in order to become a proactive agent of change in the place where they work. Generally, health science students at the 
university level in Peru start as adolescents and continue into their later teenage years, a time when self-concept and behavior are developing with a relation between the two. Additionally, university students often do not eat a balanced diet, sleep an adequate number of hours each night, or do enough exercise during the week. This problem is compounded when they have a heavy course load, as is often the case for students in the health sciences. With this in mind, the objective of this study was to identify the influence of self-concept on lifestyles in students of a private university in eastern Lima. The study has a non-experimental design and it is a quantitative study with an explanatory-causal and cross-sectional approach. The sampling method used was non-probabilistic, and 135 students participated in the study, meeting the inclusion and exclusion criteria. Their ages ranged from 16 to 24 years old (M $=20$ ) and the majority of the participants were female $(87 \%)$. The study was approved by the Ethics Committee of the university. Two previously validated instruments were chosen to measure the primary variables of the study. The Lifestyle Questionnaire (PEPS-I), consisting of 48 questions with answers on a Likert scale, consists of six factors and was chosen since it has already been validated with adolescents in Peru. The AF-5 has 30 questions grouped into five factors, and it was chosen for the self-concept variable since it has already been validated in a sample of university students in Peru. Both instruments were administered with the support of the Dean of the Faculty of Health Sciences of the university where the study was carried out. Teachers of different courses granted around 20 minutes of their class time for the study team members to come in, present the study, ask for informed consent, and then apply the instruments with those who were willing to participate. In the descriptive analysis, the average of the lifestyle profile corresponds to the self-realization dimension (43.2), followed by the health responsibility dimension (26.1) and the lowest average belongs to the exercise dimension (12.4). In the analysis of the self-con- cept variable, the social self-concept obtained the highest average (43.2) and the physical self-concept presented the lowest average (28.2). A model was developed to explore the relationships between the two variables and within the factors of each variable. This model shows that self-concept significantly influences the lifestyle of these students $(\beta=.80 ; p<.05)$, that is, self-concept is able to explain $80 \%$ of the lifestyle profile that the students currently hold. Within each variable, most of the factors for self-concept had values equal to or greater than .80 , except for the family factor which had a value of .55 while the factors for lifestyles were all above .75 except for the exercise factor.

Health science education at the university level should include integral formation not only about knowledge that can be used to treat future patients, but also about lifestyle changes that can help their own health. Self-concept and lifestyle are both in a process of formation during the university years, and they are related. Simply learning about the benefits of healthy lifestyles is not enough, as many health science students are taught these concepts but yet do not put them into practice. Social self-concept in particular, since it was the highest score among the related factors, could be a key way to encourage health science students to take what they learn and put it into practice in their own lives.

Keywords: Self-concept, healthy lifestyle, students nursing.

\section{Introducción}

Los estilos de vida saludables se refieren a aquellas conductas o comportamientos, tanto individuales como colectivas, con los cuales el individuo alcanza el bienestar, no solo físico sino también emocional (Bastías y Stiepovich, 2014; Mora y Múnera, 2015). Sin embargo, la alta frecuencia del sedentarismo, con sus riesgos sanitarios, es alarmante, dado los extensos y acelerados cambios del estilo de vida en zonas urbanas que fomentan la comodidad y el esfuerzo mínimo en todos los 
ámbitos de la vida (Alvarez, Castaño, Marín, Navas y Noreña, 2007). En un estudio realizado en Brasil, la prevalencia del sedentarismo fue extremadamente alta en la población de adolescentes, tanto en escuelas públicas como privadas. Además, el sexo femenino se asoció directamente con el sedentarismo (Nogueira et al., 2016). Por otra parte, la etapa universitaria conlleva una serie de cambios emocionales, fisiológicos y ambientales que van a determinar unas costumbres y hábitos de riesgo que, en muchos casos, serán mantenidos a lo largo de la vida y repercutirán en su estado de salud (Gallardo-Escudero et al., 2014). En relación con la importancia de los estilos de vida de los estudiantes, Sánchez-Ojeda y De Luna-Bertos (2015) indican que los jóvenes universitarios, en general, no tienen buenos hábitos alimentarios. La práctica del ejercicio físico es nula, aun cuando junto a una buena alimentación tiene efectos beneficiosos sobre la salud y un impacto positivo sobre el autoconcepto físico y el autoconcepto académico.

Un estudio realizado en Colombia identificó que el abandono del domicilio familiar, la interacción con personas de diferentes sitios de origen, el cambio en hábitos nutricionales, las actividades académicas y los patrones de sueño y descanso son factores que interfieren en el intento por mantener una vida saludable (Suescún et al., 2017). Frente a ello, las universidades y demás instituciones educativas deben involucrar programas de intervención para promover estilos de vida saludables, enfocados en la realidad de los jóvenes, para hacerlos más efectivos y así lograr que no se presenten problemas de salud en sus estudiantes (Tamayo et al., 2015).

Es importante señalar que un estilo de vida saludable, bajo un enfoque socioeducativo, no se limita a una conducta exterior, sino que implica una forma de conocer, de aprender, de adueñarse del conocimiento $\mathrm{y}$, en último término, de la realidad aparente. En el mismo sentido, la educación para la salud tiene como finalidad analizar el significado y la evolución de los acontecimientos en este campo, al subrayar aquellos elementos que influyen en ella positiva o negativamente (Muñoz, et al., 2010). Por tanto, debe hacerse un asesoramiento personalizado que priorice la conducta a cambiar según el riesgo, pero también según la disposición al cambio de la persona (Córdoba et al., 2016).

En este contexto, el autoconcepto es uno de los factores que permite al ser humano gozar de salud y bienestar emocional durante su desarrollo evolutivo (Carranza y Bermúdez-Jaimes, 2017). Asimismo, el autoconcepto es la percepción que tiene la persona de sí misma, en base a la observación de sus capacidades y limitaciones que le permite tener un sentido de la propia identidad (García y Musitu, 2001).

Existe una relación entre las dimensiones del autoconcepto y las conductas de salud en la adolescencia, ya que presentan la base de uno de los motivos básicos que guían el conocimiento de sí mismo, lo que será el motivo de automejora. Pastor, Balaguer y García-Merita (2006) consideran que el motivo de automejora conduce al sujeto a elegir aquellos comportamientos que le permiten obtener mejores autovaloraciones. El conocimiento de sí mismo permitirá fortalecer su propia concepción de salud.

Por lo expuesto, el objetivo del estudio fue identificar la influencia del autoconcepto en los estilos de vida de los universitarios. En tal sentido, se espera que los resultados puedan brindar soporte empírico a las posibles intervenciones en salud a implementar en las instituciones de educación superior, basadas en el fortalecimiento de los factores psicológicos predictores, y que la experiencia pueda ser replicada en trabajos ulteriores, con igual o diferente óptica.

\section{Método}

\section{Diseño y participantes}

Este estudio tuvo un diseño no experimental, con enfoque cuantitativo, explicativo-causal y transversal. Participaron 135 estu- 
diantes de primero a quinto año de la carrera de enfermería de una universidad privada de Lima, zona este. El método de muestreo fue no probabilístico de tipo censal, es decir, a toda la población, a la que se le aplicaron los criterios de inclusión y exclusión, razón por la que se considera factible generalizar los resultados. Como criterios de inclusión se establecieron: ser estudiantes regulares, no estar participando de algún programa con apoyo médico o psicológico y aceptar participar voluntariamente del estudio.

Las edades de los estudiantes oscilaron entre 16 y 24 años, con una media de 20 años. El $64 \%$ vivía con ambos padres, el $30 \%$ trabajaba y estudiaba. El $87 \%$ era de género femenino y el $13 \%$, masculino.

\section{Instrumentos}

La variable estilos de vida fue analizada con el Cuestionario de estilos de vida (PEPS-I; Pender, 1996). La confiabilidad del instrumento reportó un coeficiente alfa de Cronbach en un rango de .79 a .87 para las subescalas y .94 para la escala total. Se trata de un cuestionario que evalúa los estilos de vida y mide cuantitativamente el estilo de vida saludable de la persona. Contiene seis subescalas: nutrición, ejercicio, responsabilidad en salud, manejo del estrés, soporte interpersonal y autoactualización. Está conformado por 48 reactivos tipo Likert con solo un patrón de respuesta. El valor mínimo es 48 y el máximo 144 , y un mayor puntaje implica un mejor estilo de vida. Con respecto a sus propiedades psicométricas en Perú, muestra adecuados niveles de fiabilidad en las subescalas y en la escala general (>.70). Además, se muestra estable a través del tiempo, ya que sus índices oscilan entre .56 y .77 para las subescalas y el puntaje total. En referencia a la validez, el test demostró correlaciones ítem-test entre moderadas y elevadas, lo cual verifica su capacidad para medir y discriminar los constructos (Alanya, 2013).

La variable autoconcepto (A) fue medida con el test AF-5 (García y Musitu, 2001) que contiene 30 ítems distribuidos en cinco dimensiones: A. académico, A. social, A. familiar, A. emocional y A. físico. La confiabilidad del test en su versión original fue altamente significativa $(\alpha=.815)$ y su validez a través de la técnica de correlación demostró ser idónea ( $\mathrm{r}$ $=$.643) (García y Musitu, 2001). En Perú se identificó la confiabilidad con 861 estudiantes universitarios, lo que evidenció que la escala representa una muy buena confiabilidad (.866), ya que los valores estimados superaron el umbral del punto de corte (se considera como indicador de una buena confiabilidad .75) (Carranza y Bermúdez-Jaimes, 2017).

\section{Procedimiento}

Para el levantamiento de los datos se obtuvo la aprobación del Comité de Ética del Centro de Investigación en Salud y Alimentación de la universidad sede del estudio. Posteriormente, se coordinó con los docentes de las diferentes aulas con los cuales se consensuaron horarios. Ya en las aulas con los estudiantes, se les explicó la naturaleza y objetivos del trabajo, se resaltó el anonimato y la posibilidad de retirarse de la prueba en cuanto lo consideraran necesario. Obtenido el consentimiento informado, se procedió a la aplicación de los instrumentos, la cual tuvo una duración aproximada de 20 minutos.

\section{Análisis}

Para obtener los análisis descriptivos de las variables (media [M], desviación estándar [DE], asimetría [g1] y curtosis [g2]) se utilizó el programa SPSS 22.0 para Windows. El método de estimación utilizado para el modelo explicativo fue el de máxima verosimilitud (ML) que, aunque supone la normalidad multivariada, es razonablemente robusto a su incumplimiento (Curran et al., 1996), y el posible sesgo en la estimación se produciría, en todo caso, presentando un ajuste más deficiente del real (Tomás y Oliver, 1998). 
Para evaluar el ajuste del modelo, se empleó el índice RMSEA (Root Mean Square Error of Approximation), en cuyo caso, los valores menores a .05 indican buen ajuste, y entre .05 y .08 se considera aceptable (Kline, 2015). También se utilizó el índice CFI (Comparative Fit Index) y TLI (Tucker-Lewis Index) para casos con valores mayores a .95, que indican buen ajuste, y los mayores a .90 como un ajuste aceptable (Schumacker y Lomax, 2015). En todos los casos se utilizó el entorno de R Studio (R Studio Team, 2018) para R (R Core Team, 2019).

\section{Resultados}

\section{Análisis descriptivo}

La tabla 1 presenta los estadísticos descriptivos para cada una de las variables observadas en el modelo explicativo. Se aprecia que el promedio más alto en el perfil de estilos de vida corresponde a la variable autoactualización (43.2), seguido de la variable responsabilidad en la salud (26.1). Por su parte, el promedio más bajo pertenece a la variable ejercicio (12.4). En relación con las variables observadas de autoconcepto, el promedio más alto pertenece al autoconcepto social (43.2) y el más bajo al autoconcepto físico (28.2). Respecto a la asimetría y la curtosis, todos los valores están dentro de los límites esperados (entre +/- 1.5).

Tabla 1

Análisis descriptivo de las variables.

\begin{tabular}{c|c|c|c|c|c|c} 
Variables & M & DE & Min & Max & g1 & g2 \\
\hline Estilos de vida & & & & & & \\
\hline Nutrición & 16.9 & 3.5 & 6 & 24 & -.31 & -.01 \\
Ejercicio & 12.4 & 3.1 & 5 & 20 & .29 & -.04 \\
Responsabilidad en la salud & 26.1 & 4.9 & 10 & 40 & -.23 & .42 \\
Manejo del estrés & 18.6 & 3.7 & 7 & 28 & -.27 & .63 \\
Soporte interpersonal & 21.6 & 4.3 & 6 & 28 & -1.08 & 1.49 \\
Autoactualización & 43.2 & 7.2 & 13 & 52 & -1.41 & 2.93 \\
\hline Autoconcepto & & & & & & \\
\hline Académico & 38.2 & 6.3 & 14 & 50 & -.94 & 1.54 \\
Social & 43.2 & 6.8 & 18 & 56 & -1.00 & 1.43 \\
Emocional & 37.2 & 5.5 & 14 & 48 & -1.20 & 2.53 \\
Familiar & 31.3 & 5.5 & 12 & 14 & -.76 & .24 \\
Físico & 28.2 & 5.2 & 7 & 35 & -1.11 & 1.80
\end{tabular}

\section{Modelo explicativo}

En la figura 1 se aprecia que el autoconcepto influye significativamente sobre el estilo de vida de los estudiantes $(\beta=.80 ; p<.05)$, es decir, el autoconcepto logra explicar el 80 $\%$ del perfil de estilo de vida que llevan los estudiantes actualmente. En relación con los modelos de medida de las variables latentes, se observa que todos son adecuados y son representados adecuadamente por sus indicadores. Finalmente, el modelo planteado evidencia un buen ajuste a los datos (CFI $=.97$; TLI $=.95$; $\mathrm{NFI}=.93 ; \mathrm{RMSEA}=.07[\mathrm{IC} 90 \% .04-.10])$. 




Figura 1. Modelo explicativo causal de la variable estilos de vida en la variable autoconcepto.

\section{Discusión}

El estudio del autoconcepto y los estilos de vida en los estudiantes es una necesidad en el logro de la calidad del proceso de enseñanza aprendizaje. Es así que a continuación se presentan algunas experiencias previas.

En un estudio realizado en San Sebastián, España, se encontró relación entre las variables estudiadas, por lo que se dedujo que era necesario identificar cuáles eran los mecanismos psicológicos que se asocian con el mantenimiento de un estilo saludable de vida, llegando a la conclusión de que uno de esos mecanismos es el autoconcepto físico. Una vida poco saludable conlleva una percepción negativa, mientras que el autoconcepto positivo va de la mano de los hábitos sanos (Rodriguez, Goñi y Ruiz de Azúa, 2006). Estos resultados son similares a los encontrados en el presente estudio, en el que un mejor auto- concepto predice un mejor estilo de vida en los universitarios.

Con respecto a los estilos de vida, varios autores proponen diferente número de dimensiones. Un estudio realizado en Bucaramanga, Colombia, propone cuatro dimensiones del constructo estilos de vida: ejercicio y actividad física, consumo de alcohol, consumo de cigarrillo y sustancias psicoactivas, y sexualidad. La investigación concluyó que, acorde con estudios previos, el conocimiento no es suficiente para la adopción de conductas saludables, así como tampoco lo son el disponer de creencias adecuadas sobre la salud o las motivaciones consistentes frente a la práctica saludable (Arguello, Bautista, Carvajal, De Castro, Díaz y Escobar, 2009). En el presente estudio se determinó que los conocimientos sobre el cuidado de la salud obtenidos en autoactualización y responsabilidad en salud tienen el más alto promedio, lo que significaría 
que no siempre es suficiente el conocimiento per se si no va acompañado de una decisión de un cambio en las prácticas de estilos de vida saludables.

En un trabajo de investigación realizado en los colegios de Chía, Colombia, Díaz-Martínez, Tovar, y Torres (2015) encontraron que la mayoría de los participantes estudiados mostró una autoimagen (autoconcepto) moderadamente alta y media, lo cual podría favorecer el desarrollo de estilos de vida saludables. En el presente estudio se encontró que el autoconcepto social es más alto que el autoconcepto físico, posiblemente porque los estudiantes de enfermería desarrollan la empatía y la comunicación en el servicio con el cuidado que otorgan a las personas, aspecto relacionado con el intercambio social con las personas que los rodean (Marques, Peralta, Santos, Martins y Gaspar de Matos, 2019).

Existen importantes iniciativas para mejorar los estilos de vida de los estudiantes, como es el caso de Yaguajay (Cuba), donde la universidad desarrolla estrategias para incentivar una cultura saludable, unificar esfuerzos, coordinar acciones, involucrar sectores, consolidar alianzas interinstitucionales dentro y fuera de la esfera sanitaria, con lo que se demostró la fortaleza de la promoción de salud dentro de los procesos de desarrollo local (Reyes, 2018). La universidad de donde proceden los estudiantes del presente estudio tiene programas de fortalecimiento del estilo de vida, lo que significa que los resultados servirán para identificar aspectos a ser mejorados.

Los comportamientos adolescentes, que se caracterizan por la búsqueda de una nueva identidad y el aumento de la independencia, es foco de interés y preocupación de las familias, los educadores y los políticos. Sin embargo, ninguna de las medidas que se propongan para potenciar estilos de vida saludables entre los adolescentes (por ejemplo, para reducir el consumo de alcohol) será efectiva por sí misma si solo se considera el conflicto de orden público. A este hay que unir el conflicto de salud y tener muy presentes las medidas de prevención antes de las medidas de acción (Hernando, Oliva y Pertegal, 2013). En este contexto, las medidas que se pueden derivar del presente estudio fortalecerán los estilos de vida, así como el autoconcepto de los estudiantes.

Dado que la adolescencia es una fase de grandes cambios en las distintas dimensiones de la vida, puede ser especialmente interesante el estudio y la intervención sobre estas variables para la configuración de estilos de vida saludables que promuevan el desarrollo positivo (Dias, Bastos, Marzo y García del Castillo, 2016). De hecho, los estilos de vida saludables cultivados en la adolescencia y adultez temprana tendrán efectos positivos en la vida futura de la persona y ese es el reto en la formación de profesionales: preparar a personas con calidad de vida que sean agentes proactivos y modelos de hábitos saludables.

El hecho de encontrar que un alto porcentaje de estudiantes tenga un promedio más alto en la variable autoactualización y responsabilidad en salud podría estar relacionado con el autocuidado de su salud basado en el conocimiento que van adquiriendo como estudiantes. Los planes de estudio de la mayoría de las universidades tienen asignaturas $o$ módulos al respecto. La educación actual ha dado un impulso en este aspecto y, por ello, es necesario generar estudios de investigación que permitan conocer cómo los estudiantes cuidan su salud.

En muchos casos los jóvenes universitarios poseen una alta presencia de creencias favorables sobre hábitos de vida saludables y, a pesar de ello, no lo llevan a la práctica. Es así que, muchas veces, los conocimientos no están directamente relacionados con los hábitos. Sanchez-Ojeda y De Luna-Bertos (2015) encontraron que la práctica del ejercicio físico es nula, aun sabiendo que tiene efectos beneficiosos sobre la salud. Los participantes del estudio no practican ejercicios físicos necesarios para su salud, o no los hacen de manera programada, aunque 
conocen sus ventajas. En el presente estudio se observó una situación similar, es decir, los estudiantes no practicaban ejercicios físicos de manera programada, aunque conocían sus ventajas. Este hecho puede estar relacionado con el limitado tiempo que tienen para dicha actividad, debido a la gran distancia entre el lugar de estudios y los centros de prácticas y el tráfico congestionado casi a toda hora, por lo que se puede pensar que habría una variable interviniente en el cumplimiento de la actividad física.

El autoconcepto social fue el que obtuvo mayor puntaje, que difiere del autoconcepto físico que obtuvo la menor puntuación, tema que puede ser analizado y abordado para fortalecer la autoestima y, por ende, el autoconcepto.

El estudio permite reflexionar sobre una problemática presente en las universidades, ya que el autoconcepto de los estudiantes es un factor clave para lograr un estilo de vida saludable, que les permita desenvolverse como estudiantes y, posteriormente, como profesionales. Esto también permitiría que se constituyan en agentes proactivos de cambio en la búsqueda del mejoramiento de la calidad de vida de las personas a las que atenderá y no solo por teoría, sino con el ejemplo. Además, los resultados del presente estudio podrán servir de base para realizar estudios posteriores desde diferentes ópticas.

\section{Conclusiones}

El autoconcepto influye significativamente sobre el estilo de vida de los estudiantes $(\beta=$ $.80 ; \mathrm{p}<.05)$, es decir, el autoconcepto logra explicar el $80 \%$ del perfil de estilo de vida que llevan los estudiantes actualmente.

\section{Referencias}

Alanya, J. L. (2013). Validez y confiabilidad del cuestionario de estilo de vida promotor de salud (PEPS - I) en adolescentes [Universidad Nacional Federico Villarreal-Lima,
Perú]. http://repositorio.unfv.edu.pe/handle/ $\mathrm{UNFV} / 1076$

Alvarez, J. L., Castaño, J. J., Marín, J. G., Navas, C. y Noreña, P., et al. (2007). Estilos de vida en estudiantes de la Universidad de Manizales, año 2006. Archivos de Medicina, 1(15), 46-56. https://doi.org/10.30554/ archmed.15.0.1504.2007

Arguello, M., Bautista, Y., Carvajal, J., De Castro, K., Díaz, D., Escobar, M., et al. (2009). Estilos de vida en estudiantes del área de la salud de Bucaramanga. Revista de Psicología: Universidad de Antioquia, 1(2), 27-42. https://

revistas.udea.edu.co/index.php/psicologia/ article/view/10019

Carranza, R. y Bermúdez-Jaimes, M. (2017). Análisis psicométrico de la escala de autoconcepto AF5 de García y Musitu en estudiates universitarios de Tarapoto (Perú). Interdisciplinaria, 34(2), 459-472. https://doi. org/10.16888/interd.2017.34.2.13

Córdoba, R., Camarelles, F., Muñoz, E., Gómez, J., Ramírez, J., San José, J., et al. (2016). Recomendaciones sobre el estilo de vida. Atención Primaria, 48(Supl 1), 27-38. https://doi. org/10.1016/S0212-6567(16)30186-X

Curran, P. J., West, S. y Finch, J. F. (1996). La robustez de las estadísticas de prueba ante la no normalidad y el error de especificación en el análisis factorial confirmatorio. Métodos Psicológicos, 1(1), 16-29. https:// asu.pure.elsevier.com/en/publications/the-ro-

bustness-of-test-statistics-to-nonnormality-and-specificati

Dias, P. C., Bastos, A. S., Marzo, J. C. y García del Castillo, J. C. (2016). Bienestar, calidad de vida y regulación afectiva en adolescentes portugueses. Atención Primaria, 48(7), 432-439. https://doi.org/10.1016/j.aprim.2015.09.005

Díaz-Martínez, M., Tovar M. B. y Torres A. M. (2015). Estilos de vida y autoimagen en estudiantes de colegios oficiales en Chía, Cundinamarca. Pediatría, 48(1), 15-20. https://doi. org/10.1016/j.rcpe.2015.03.001

Gallardo-Escudero, A., Muñoz, M. J. y Planells del Pozo, E.M. (2014). La etapa universitaria no favorece el estilo de vida saludable en las 
estudiantes granadinas. Nutrición Hospitalaria, 31(2), 975-979. https://doi.org/10.3305/ nh.2015.31.2.8303

García, F. y Musitu, G. (2001). AF5 Autoconcepto Forma 5. TEA.

Hernando, A., Oliva A. y Pertegal, M. A. (2013). Diferencias de género en los estilos de vida de los adolescentes. Psychosocial Intervention, 22(1), 15-23. https://doi.org/10.5093/in2013a3

Kline, R. B. (2015). Principles and Practice of Structural Equation Modeling (4th ed.). The Guilford Press.

Marques, A., Peralta, M., Santos, T., Martins J. y Gaspar de Matos M. (2019). La salud autoevaluada y la calidad de vida relacionada con la salud están relacionadas con el estilo de vida saludable de los adolescentes. Salud Pública, 170(mayo), 89-94. https://doi.org/10.1016/j. puhe.2019.02.022

Mora, S. L. y Múnera, F. A. (2015). Evaluación de estilos de vida saludable en la Facultad de Medicina de la Fundación Universitaria de Ciencias de la Salud. Repertorio de Medicina y Cirugía, 24(4), 267-274. https://www. fucsalud.edu.co/sites/default/files/2017-01/ EVALUACIÓN DE ESTILOS DE VIDA.pdf

Muñoz, J., Maldonado T. J. y Albarrán J. (2010). Competencias para la promoción del estilo de vida. Horizonte Sanitario, 6(3), 4-11. http://www.redalyc.org/articulo. oa? $\mathrm{id}=457845050001$

Nogueira, F. M., Veiga, T. V., Gondim, M. R., de Souza, C., Mendoza, C. L., Rolim, T. I., Lima, A. L., Sebba, W. K. y Veiga, P. C. (2016). Estilo de vida sedentario y sus asociadosfactores entre los adolescentes del público yescuelas privadas de la capital de un estado brasileño. BMC Public Health, 16(1), 1-8. https://doi. org/10.1186/s12889-016-3836-9

Pastor, Y., Balaguer I. y García-Merita M. (2006). Relaciones entre el autoconcepto y el estilo de vida saludable en la adolescencia media: un modelo exploratorio. Psicothema, 18(1), 18-24. http://www.psicothema.com/psicothema.asp? $\mathrm{id}=3170$

Pender, N. (1996). Cuestionario de Perfil de Estilo de Vida. 42-44. http://catarina.udlap. $\mathrm{mx} / \mathrm{u}$ dl_a/tales/documentos/lps/hernandez_d_md/ apendiceA.pdf

Reyes, R. M. (2018). La universidad y su articulación con la sociedad en la búsqueda de entornos saludables en Yaguajay. Humanidades Médicas, 18(3), 576-597. http:// scielo.sld.cu/scielo.php?script=sci_arttext\&pid $=$ S1727-81202018000300576

Rodriguez, A., Goñi A. y Ruiz de Azúa S. (2006). Autoconcepto físico y estilos de vida en la adolescencia. Intervención Psicosocial, 15(1), 81-94. http://www.redalyc.org/articulo. oa? id=179814011006

Sanchez-Ojeda, M.A. y De Luna-Bertos E. (2015). Hábitos de vida saludable en la población universitaria. Nutrición Hospitalaria, 31(5), 1910-1919. https://doi.org/10.3305/ nh.2015.31.5.8608

Schumacker, R. E. y Lomax, R. G. (2015). A Beginner's Guide to Structural Equation Modeling: Fourth Edition (4th ed.). Routledge. Suescún, S. E., Sandoval, C., Hernández, F., Araque, I., Fagua, L. H., Bernal, F. y Corredor, S. P. (2017). Estilos de vida en estudiantes de Boyaca, Colombia. Revista de la Facultad de Medicina, 65(2), 227-232. https://doi. org/10.15446/revfacmed.v65n2.58640

Tamayo, J. A., Rodríguez, K., Escobar K. y Mejía A. M. (2015). Estilos de vida de estudiantes de odontología. Hacia La Promoción de La Salud, 20(2), 147-160. https://doi.org/10.17151/ hpsal.2015.20.2.10

Tomás, J. M. y Oliver, A. (1998). Efectos de formato de respuesta y método de estimación en análisis factorial confirmatorio. Psicothema, 10(1), 197-208. http://www.psicothema.com/ pdf/155.pdf

Recibido: 27 de enero de 2020 Aceptado: 29 de septiembre de 2021 